\title{
Quantification of Metabolic Rearrangements During Neural Stem Cells Differentiation into Astrocytes by Metabolic Flux Analysis
}

\author{
João V. Sá ${ }^{1,2} \cdot$ Susanne Kleiderman $^{3} \cdot$ Catarina Brito $^{1,2} \cdot$ Ursula Sonnewald $^{4,5}$. \\ Marcel Leist ${ }^{3} \cdot$ Ana P. Teixeira ${ }^{1,2} \cdot$ Paula M. Alves ${ }^{1,2}$
}

\begin{abstract}
Proliferation and differentiation of neural stem cells (NSCs) have a crucial role to ensure neurogenesis and gliogenesis in the mammalian brain throughout life. As there is growing evidence for the significance of metabolism in regulating cell fate, knowledge on the metabolic programs in NSCs and how they evolve during differentiation into somatic cells may provide novel therapeutic approaches to address brain diseases. In this work, we applied a quantitative analysis to assess how the central carbon metabolism evolves upon differentiation of NSCs into astrocytes. Murine embryonic stem cell (mESC)-
\end{abstract}

\section{S.I. : In honor of Dr. Mary McKenna.}

Ana P. Teixeira anat@ibet.pt

$\triangle$ Paula M. Alves marques@ibet.pt

1 IBET, Instituto de Biologia Experimental e Tecnológica, Apartado 12, 2780-901 Oeiras, Portugal

2 Instituto de Tecnologia Química e Biológica António Xavier, Universidade Nova de Lisboa, Avenida da República, 2780-157 Oeiras, Portugal

3 The Doerenkamp-Zbinden Chair of in-vitro Toxicology and Biomedicine/Alternatives to Animal Experimentation, University of Konstanz, Konstanz, Germany

4 Department of Neuroscience, Faculty of Medicine, Norwegian University of Science and Technology, 7491 Trondheim, Norway

5 Department of Drug Design and Pharmacology Faculty of Health and Medical Sciences, University of Copenhagen, 2100 Copenhagen, Denmark derived NSCs and astrocytes were incubated with labelled $\left[1-{ }^{13} \mathrm{C}\right]$ glucose and the label incorporation into intracellular metabolites was followed by GC-MS. The obtained ${ }^{13} \mathrm{C}$ labelling patterns, together with uptake/secretion rates determined from supernatant analysis, were integrated into an isotopic non-stationary metabolic flux analysis $\left({ }^{13} \mathrm{C}\right.$ MFA) model to estimate intracellular flux maps. Significant metabolic differences between NSCs and astrocytes were identified, with a general downregulation of central carbon metabolism during astrocytic differentiation. While glucose uptake was 1.7-fold higher in NSCs (on a per cell basis), a high lactate-secreting phenotype was common to both cell types. Furthermore, NSCs consumed glutamine from the medium; the highly active reductive carboxylation of alpha-ketoglutarate indicates that this was converted to citrate and used for biosynthetic purposes. In astrocytes, pyruvate entered the TCA cycle mostly through pyruvate carboxylase (81\%). This pathway supported glutamine and citrate secretion, recapitulating well described metabolic features of these cells in vivo. Overall, this fluxomics study allowed us to quantify the metabolic rewiring accompanying astrocytic lineage specification from NSCs.

Keywords Neural stem cells - Astrocytic differentiation . Metabolic flux analysis · Carbon labelling cultures

\section{Introduction}

Neural stem cells (NSCs) are multipotent cells present in the developing brain that persist in restricted regions of postnatal and adult brains, where they continue to produce the three neural lineages: neurons, astrocytes and oligodendrocytes [18]. Astrocytes are the most abundant brain cells [22], being involved in virtually every function of the 
central nervous system, including energy metabolism [28], ionic homeostasis [32] and synaptic transmission [15, 33]. Importantly, glutamatergic and GABAergic neurotransmission processes are dependent on astrocytic metabolism for neurotransmitter replenishment [4]. Furthermore, astrocytes store brain energy currency in the form of glycogen [7], and release substrates for neuronal oxidative phosphorylation [25]. Moreover, astrocytes have been suggested as contributors to neurodegenerative disorders by various mechanisms, including via metabolic dysfunction [27], making them potential targets for novel strategies to treat brain disorders. Given their importance for brain function, the generation of astrocytes from NSCs has attracted much attention recently. It is known that NSCs and astrocytes share several phenotypic and functional features [12, 31], but their metabolic programs have not been compared at a global metabolic flux level yet.

In vivo intracellular metabolic fluxes have been estimated using several mathematical frameworks. When metabolic steady-state can be assumed (i.e all fluxes and intracellular concentrations are approximately constant over time), ${ }^{13} \mathrm{C}$-Metabolic flux analysis $\left({ }^{13} \mathrm{C}-\mathrm{MFA}\right)$ is the most useful tool to provide a metabolic snapshot of cells [29, 30, 38]. Nuclear magnetic resonance (NMR) spectroscopy and/or mass spectrometry (MS) techniques are used to measure the incorporation of a ${ }^{13} \mathrm{C}$ label from medium nutrients into intracellular metabolites. A computational framework based on both metabolite and isotopomer balances is then used to integrate this data with experimentally determined uptake and secretion rates to estimate intracellular metabolic fluxes. This approach has been applied to study metabolic network operation and diagnosing of phenotypic perturbations in several biological systems [1, 10, 20, 21], including primary astrocytic cultures [2].

In this work, we present the first quantitative study of the metabolic programs during specification of astrocytes from NSCs. To obtain highly homogeneous non-proliferating astrocyte cultures, we have recently developed a protocol using pure and defined populations of mouse pluripotent stem cell-derived NSCs [16]. Exposure to BMP4 promoted astrocytic differentiation of these NSCs within 2 days. The resulting astrocytes expressed several positive astrocyte markers (GFAP, aquaporin-4, and GLT1), showed negligible expression of the NSC marker nestin, and acquired innate immune functions [16]. Herein, NSCs and NSC-derived astrocytes were incubated with $\left[1-{ }^{13} \mathrm{C}\right]$ glucose, and the transient ${ }^{13} \mathrm{C}$ labelling profiles of intracellular metabolites were modelled by isotopically non-stationary ${ }^{13} \mathrm{C}-\mathrm{MFA} \quad$ [23], identifying significantly different metabolic flux maps in each population.

\section{Materials and Methods}

\section{Differentiation of NSCs and Astrocytes from Murine Embryonic Stem Cells}

The murine embryonic stem cell (mESC) line CGR8 (07032901, Sigma) was used in this work for commitment to the neural lineage, as described in [16]. Briefly, for differentiation into neural stem cells (NSCs), cultures of mESCs were harvested with $0.05 \%$ trypsin, pelleted, and replated in N2B27-medium (1:1 Neurobasal-A and Advanced DMEM/F12, containing N2 \& B27 supplements, $100 \mu \mathrm{M} \beta$-mercaptoethanol, $7.5 \mu \mathrm{g} / \mathrm{mL}$ insulin, and $50 \mu \mathrm{g} /$ $\mathrm{mL}$ bovine serum albumin). On day 7 , the growth factors FGF2 (20 ng/mL) and EGF (20 ng/mL) were added. After about 8 to 12 passages of NSCs, homogeneous and aggregate-free cultures were obtained and used for differentiation into astrocytes using N2B27-medium supplemented with BMP4 $(20 \mathrm{ng} / \mathrm{mL})$. Cells were incubated at 37 ${ }^{\circ} \mathrm{C}$ with $5 \% \mathrm{CO}_{2}$.

\section{Stable Isotope Cultures}

For isotopic labelling, NSCs and astrocytes were cultured in 6-well plates with custom N2B27-medium without glucose and without glutamine, supplemented with $10 \mathrm{mM}$ $\left[1-{ }^{13} \mathrm{C}\right]$ glucose (Sigma-Aldrich, 297046) and $2 \mathrm{mM}$ glutamine for NSCs, or without glutamine supplementation for astrocytes.

Sampling was performed immediately after label administration and then at $3 \mathrm{~h}, 12 \mathrm{~h}$, and $24 \mathrm{~h}$ (Figure 1a). Replicate samples of culture supernatants were collected, clarified $(200 \times g$ for $10 \mathrm{~min})$ and stored at $-20{ }^{\circ} \mathrm{C}$ for later extracellular metabolite analysis (see below). Upon removing the culture supernatants, cell monolayers were washed twice with ice-cold PBS to eliminate tracer amounts of extracellular metabolites, and the plates were placed on liquid nitrogen to rapidly stop metabolism. For intracellular metabolite extraction, $700 \mu \mathrm{l}$ of ethanol $70 \%$ $(\mathrm{v} / \mathrm{v})$ were added to each well and the cells were detached using a cell scraper, followed by clarification $(15 \mathrm{~min}$ at $20,000 \times g$ ) and storage of the supernatants at $-80{ }^{\circ} \mathrm{C}$ until GC-MS analysis (see below). The pellets containing cellular material were stored at $-20{ }^{\circ} \mathrm{C}$ for protein quantification (see below). Additional wells seeded with NSCs and astrocytes were used to profile cell concentration and viability along the same sampling schedule.

\section{Analysis of Extracellular Metabolites}

Glucose and lactate concentrations in culture supernatants were measured with an automated YSI 7100 Multiparameter 
Bioanalytical System (Dayton, OH, USA). Amino acid concentrations were analyzed by HPLC using the Waters AccQ.Tag Amino Acid Analysis Method (Waters, Milford, MA) as described elsewhere [9]. The extracellular concentrations of citrate and pyruvate were determined by ${ }^{1} \mathrm{H}-\mathrm{NMR}$ spectroscopy using a $500 \mathrm{MHz}$ Avance spectrometer (Bruker, Billerica, MA) with a $5 \mathrm{~mm}$ QXI inversed probe. Spectra were recorded at $25{ }^{\circ} \mathrm{C}$ using a NOESY-based pulse sequence with water pre-saturation, performing 256 scans with $4 \mathrm{~s}$ acquisition time, $2 \mathrm{~s}$ relaxation delay and $100 \mathrm{~ms}$ mixing time. DSS-d6 (Sigma Aldrich, St. Louis, MO; USA) was used as internal standard for metabolite quantification in all samples. In order to obtain a similar $\mathrm{pH}$ between samples, they were mixed with phosphate buffer ( $\mathrm{pH}$ 7.4) prepared in $\mathrm{D}_{2} \mathrm{O}$ at a 2:1 ratio. Before spectra acquisition, the spectrometer was calibrated by determining the $90^{\circ}$ pulse and the water chemical shift centre of each sample. Each spectrum was phased, baseline corrected and integrated using the Chenomx NMR Suite 8.0 (Chenomx Inc., Edmonton, Alberta, Canada) software.

\section{Cell Concentration, Viability and Protein Content Measurements}

Total cell concentration was determined after trypsinization using the haemocytometer counting method. Cell viability was measured by quantification of resazurin reduction. Resazurin was added to the medium at $1 \mu \mathrm{g} / \mathrm{ml}$ for $30 \mathrm{~min}$ at $37{ }^{\circ} \mathrm{C}$. Resazurin reduction $(560 \mathrm{~nm}$ excitation, $590 \mathrm{~nm}$ emission) was measured as cell viability parameter using the Infinite ${ }^{\circledR} 200$ PRO multimode reader (Tecan). The protein content was measured in cell pellets after metabolite extraction. The cell pellets were lysed in $2 \%$ sodium dodecyl sulfate (SDS) (in $125 \mathrm{mM}$ Trizma, $10 \%$ glycerol) and the protein quantified with the Pierce ${ }^{\mathrm{TM}}$ BCA Protein Assay Kit (Thermo Scientific), according to the manufacturer's protocol.

\section{Quantification of Intracellular Mass Isotopomer Distributions by GC-MS}

For analysis of ${ }^{13} \mathrm{C}$ enrichment in lactate, amino acids (alanine, aspartate, glutamate, and glutamine), and TCA cycle intermediates (citrate, fumarate, succinate and malate), lyophilized metabolite extracts were dissolved in $0.01 \mathrm{M}$ $\mathrm{HCl}$ followed by $\mathrm{pH}$ adjustment to $\mathrm{pH}<2$ with $6 \mathrm{M} \mathrm{HCl}$. The samples were then dried under atmospheric air $\left(50{ }^{\circ} \mathrm{C}\right)$, and the metabolites extracted in multiple steps into an organic phase of ethanol and benzene. Afterwards, the samples were lyophilized again and derivatized with N-Methyl-N-(tButyldimethylsilyl)trifluoroacetamide (MTBSTFA) $+1 \%$ t-butyldimethylchlorosilane (TBDMSCI). The glycolytic intermediates phosphoenolpyruvate (PEP) and 3-phosphoglycerate
(3PG) were analyzed using a similar protocol to that described in [14]. Briefly, lyophilized metabolite extracts were dissolved in methanol and dried under atmospheric air $\left(50{ }^{\circ} \mathrm{C}\right)$. The metabolites were then extracted with toluene and dried again under atmospheric air $\left(50^{\circ} \mathrm{C}\right)$. Derivatization was performed using a mixture of N-Methyl$\mathrm{N}$-(trimethylsilyl)trifluoroacetamide (MSTFA) $+1 \%$ trimethylchlorosilane and acetonitrile.

The mass isotopomer distributions of the derivatized metabolites were analyzed on an Agilent 6890 gas chromatographer equipped with a capillary column (WCOT fused silica $25 \mathrm{~mm} \times 0.25 \mathrm{~mm}$ ID, $0.25 \mu \mathrm{m}$ film thickness, VF-1ms, Varian), and connected to an Agilent 5975B mass spectrometer with electron impact ionization (Agilent Technologies, Palo Alto, CA, USA). All ions (M, M1, Mm, where $\mathrm{M}$ is the mass/charge ratio of the unlabelled derivatized fragment and $\mathrm{m}$ is the number of labelled carbons) were measured by spectra integration using the MassHunter software (Agilent Technologies, Palo Alto, CA, USA) and corrected for natural ${ }^{13} \mathrm{C}$ abundance using non-enriched standards [35].

\section{Metabolic Flux Determination}

Estimation of metabolic fluxes of NSCs and astrocytes was performed by isotopic non-stationary ${ }^{13} \mathrm{C}$-MFA. Our experimental data was comprised of exchanged rates and corrected mass isotopomer distributions of intracellular metabolites. The extracellular rates were calculated by linear fitting of extracellular metabolite profiles (assuming metabolic steady state throughout the labeling experiment) and normalized to protein content per well. The reaction network used for flux estimation included the main pathways of central carbon metabolism: glycolysis, pentose phosphate pathway, tricarboxylic acid cycle and amino acids metabolism. Table 1 lists all metabolic reactions and carbon atom transitions (balanced and unbalanced metabolite pools are also identified). Since cell concentration and protein content were maintained during the labelling experiment, reactions for biomass formation were omitted in the network. Extracellular transport reactions were considered reversible to allow equilibration with extracellular pools, except for essential amino acids and glucose.

Non-stationary ${ }^{13} \mathrm{C}$-MFA was performed using the publicly available software package INCA [40], which implements the elementary metabolite units framework [41]. Briefly, fluxes are estimated by minimizing the variance-weighted sum of squared residuals (SSR) between experimental measurements and model predictions using least-squares regression. At least 10 restarts with random initial values were performed to find a global optimum [11]. At convergence, the obtained solution was subject to 
Table 1 Metabolic network model used for isotopic nonstationary ${ }^{13} \mathrm{C}$-MFA for NSCs and astrocytes, along with the carbon atom transitions

\begin{tabular}{|c|c|}
\hline & Glycolysis \\
\hline $\mathrm{R} 1$ & G6P (abcdef) $\leftrightarrow$ F6P (abcdef) \\
\hline $\mathrm{R} 2$ & F6P (abcdef) $\rightarrow$ FBP (abcdef) \\
\hline $\mathrm{R} 3$ & FBP $($ abcdef $) \leftrightarrow$ DHAP $($ cba $)+$ GAP $($ def $)$ \\
\hline $\mathrm{R} 4$ & DHAP $(a b c) \leftrightarrow$ GAP $(a b c)$ \\
\hline R5 & $\mathrm{GAP}(\mathrm{abc}) \leftrightarrow 3 \mathrm{PG}(\mathrm{abc})$ \\
\hline R6 & $3 \mathrm{PG}(\mathrm{abc}) \leftrightarrow \mathrm{PEP}(\mathrm{abc})$ \\
\hline \multirow[t]{2}{*}{$\mathrm{R} 7$} & PEP $(a b c) \rightarrow$ Pyr.c $(a b c)$ \\
\hline & Pentose-phosphate pathway \\
\hline $\mathrm{R} 8$ & G6P (abcdef) $\rightarrow$ P5P (bcdef) $+\mathrm{CO}_{2}$ (a) \\
\hline R9 & P5P $($ abcde $)+$ P5P (pqrst) $\leftrightarrow$ GAP $($ rst $)+$ S7P (pqabcde $)$ \\
\hline $\mathrm{R} 10$ & S7P (abcdefg) + GAP $(x y z) \leftrightarrow$ E4P $($ defg $)+$ F6P (abcxyz) \\
\hline \multirow[t]{2}{*}{ R11 } & $\mathrm{E} 4 \mathrm{P}(\mathrm{abcd})+\mathrm{P} 5 \mathrm{P}(\mathrm{pqrst}) \leftrightarrow \mathrm{GAP}(\mathrm{rst})+\mathrm{F} 6 \mathrm{P}(\mathrm{pqabcd})$ \\
\hline & Lactate and alanine accumulation \\
\hline $\mathrm{R} 12$ & Pyr.c $(a b c) \leftrightarrow$ Lac $(a b c)$ \\
\hline \multirow{2}{*}{$\mathrm{R} 13$} & Pyr.c $(a b c) \leftrightarrow$ Ala $(a b c)$ \\
\hline & TCA cycle and pyruvate cycling \\
\hline $\mathrm{R} 14$ & Pyr.m $(a b c) \rightarrow$ AcCoA.m $(b c)+\mathrm{CO}_{2}(a)$ \\
\hline $\mathrm{R} 15$ & Pyr.m $(\mathrm{abc})+\mathrm{CO}_{2}(\mathrm{~d}) \rightarrow$ OAC (abcd) \\
\hline R16 & $\mathrm{OAC}($ abcd $)+$ AcCoA.m $($ ef $) \rightarrow$ Cit $($ dcbfea $)$ \\
\hline $\mathrm{R} 17$ & Cit $($ abcdef $) \leftrightarrow$ AKG (abcde) $+\mathrm{CO}_{2}(\mathrm{f})$ \\
\hline $\mathrm{R} 18$ & $\mathrm{AKG}($ abcde $) \rightarrow \mathrm{SucCoA}(\mathrm{bcde})+\mathrm{CO}_{2}(\mathrm{a})$ \\
\hline R19 & SucCoA $($ abcd $) \leftrightarrow$ Suc (abcd) \\
\hline $\mathrm{R} 20$ & Suc $($ abcd $) \leftrightarrow$ Fum $($ abcd $)$ \\
\hline $\mathrm{R} 21$ & Fum $(a b c d) \leftrightarrow$ Mal $($ abcd $)$ \\
\hline $\mathrm{R} 22$ & $\mathrm{OAC}(\mathrm{abcd}) \leftrightarrow \mathrm{Mal}(\mathrm{abcd})$ \\
\hline \multirow[t]{2}{*}{$\mathrm{R} 23$} & Mal $($ abcd $) \rightarrow$ Pyr.m $(a b c)+\mathrm{CO}_{2}(d)$ \\
\hline & Lipid precursor generation \\
\hline \multirow[t]{2}{*}{$\mathrm{R} 24$} & Cit $($ dcbfea $) \rightarrow$ OAC (abcd) + AcCoA.c $(e f)$ \\
\hline & Amino acids metabolism \\
\hline $\mathrm{R} 25$ & Gln (abcde) $\leftrightarrow$ Glu (abcde) \\
\hline $\mathrm{R} 26$ & Glu (abcde) $\leftrightarrow$ AKG (abcde) \\
\hline $\mathrm{R} 27$ & Asn (abcd) $\leftrightarrow$ Asp (abcd) \\
\hline $\mathrm{R} 28$ & Asp (abcd) $\leftrightarrow$ OAC (abcd) \\
\hline $\mathrm{R} 29$ & Ser $(a b c) \rightarrow$ Pyr.c $(a b c)$ \\
\hline $\mathrm{R} 30$ & Ser $(a b c) \leftrightarrow$ Gly $(a b)+$ C1 $(c)$ \\
\hline $\mathrm{R} 31$ & Glu (abcde) $\leftrightarrow$ Pro (abcde) \\
\hline $\mathrm{R} 32$ & $\mathrm{Val}(\mathrm{abcde})+\mathrm{CO}_{2}(\mathrm{f}) \rightarrow$ Suc $($ dcef $)+\mathrm{CO}_{2}(\mathrm{a})+\mathrm{CO}_{2}(\mathrm{~b})$ \\
\hline $\mathrm{R} 33$ & $\begin{array}{l}\text { Ile (abcdef) }+\mathrm{CO}_{2}(\mathrm{~g}) \rightarrow \text { Suc (bcdg) }+ \text { AcCoA.m (ef) } \\
\quad+\mathrm{CO}_{2} \text { (a) }\end{array}$ \\
\hline R34 & $\begin{array}{l}\text { Leu (abcdef) }+\mathrm{CO}_{2}(\mathrm{~g}) \rightarrow \text { AcCoA.m }(\mathrm{bc})+\text { AcCoA.m }(\text { de }) \\
+ \text { AcCoA.m }(\mathrm{gf})+\mathrm{CO}_{2}(\mathrm{a})\end{array}$ \\
\hline $\mathrm{R} 35$ & Thr (abcd) $\rightarrow$ AcCoA.m (cd) + Gly (ab) \\
\hline R36 & Phe (abcdefghi) $\rightarrow$ Tyr (abcdefghi) \\
\hline R37 & $\begin{array}{l}\text { Tyr (abcdefghi) } \rightarrow \text { Fum }(\text { defg })+\text { AcCoA.m }(b c) \\
+ \text { AcCoA.m }(\text { hi })+\mathrm{CO}_{2}(\text { a) }\end{array}$ \\
\hline $\mathrm{R} 38$ & $\begin{array}{l}\text { Met (abcde) }+ \text { Ser (fgh) }+\mathrm{CO}_{2}(\mathrm{i}) \rightarrow \text { Suc (bcdi) }+ \text { Cys.snk } \\
\quad(\text { fgh })+\mathrm{CO}_{2} \text { (a) }+\mathrm{Cl} \text { (e) }\end{array}$ \\
\hline R39 & $\begin{array}{l}\text { Lys (abcdef) } \rightarrow \mathrm{CO}_{2}(\mathrm{a})+\mathrm{CO}_{2}(\mathrm{f})+\text { AcCoA.m }(\mathrm{bc}) \\
\quad+\text { AcCoA.m }(\mathrm{de})\end{array}$ \\
\hline $\mathrm{R} 40$ & His (abcdef) $\rightarrow$ Glu (edcba) + C1 (f) \\
\hline $\mathrm{R} 41$ & $\operatorname{Arg}($ abcdef $) \rightarrow$ Glu (abcde) + Urea.snk (f) \\
\hline
\end{tabular}


Table 1 continued

\begin{tabular}{|c|c|}
\hline R42 & $\begin{array}{l}\mathrm{Glu}(\text { abcde })+\mathrm{CO}_{2}(\mathrm{f}) \rightarrow \mathrm{Arg}(\text { abcdef }) \\
\text { Intracellular transport }\end{array}$ \\
\hline \multirow[t]{2}{*}{$\mathrm{R} 43$} & Pyr.c $(a b c) \leftrightarrow$ Pyr.m $(a b c)$ \\
\hline & Extracellular transport \\
\hline R44 & $\mathrm{CO}_{2}$ (a) $\leftrightarrow \mathrm{CO}_{2}$. ext (a) \\
\hline R45 & Glc.ext (abcdef) $\rightarrow$ G6P (abcdef) \\
\hline $\mathrm{R} 46$ & Lac $(a b c) \leftrightarrow$ Lac.ext $(a b c)$ \\
\hline R47 & Ala $(a b c) \leftrightarrow$ Ala.ext $(a b c)$ \\
\hline R48 & Pyr.ext $(a b c) \leftrightarrow$ Pyr.c (abc) \\
\hline R49 & Cit (abcdef) $\rightarrow$ Cit.ext (abcdef) \\
\hline R50 & Gln (abcde) $\leftrightarrow$ Gln.ext (abcde) \\
\hline R51 & Glu (abcde) $\leftrightarrow$ Glu.ext (abcde) \\
\hline R52 & Asp (abcd) $\leftrightarrow$ Asp.ext (abcd) \\
\hline $\mathrm{R} 53$ & Asn (abcd) $\leftrightarrow$ Asn.ext (abcd) \\
\hline R54 & Ser.ext $(a b c) \leftrightarrow$ Ser $(a b c)$ \\
\hline R55 & Gly $(a b) \leftrightarrow$ Gly.ext $(a b)$ \\
\hline R56 & Pro.ext (abcde) $\leftrightarrow$ Pro (abcde) \\
\hline R57 & Val.ext (abcde) $\rightarrow$ Val (abcde) \\
\hline R58 & Ile.ext (abcdef) $\rightarrow$ Ile (abcdef) \\
\hline R59 & Leu.ext (abcdef) $\rightarrow$ Leu (abcdef) \\
\hline R60 & Thr.ext (abcd) $\rightarrow$ Thr (abcd) \\
\hline R61 & Phe.ext (abcdefghi) $\rightarrow$ Phe (abcdefghi) \\
\hline R62 & Tyr.ext (abcdefghi) $\rightarrow$ Tyr (abcdefghi) \\
\hline R63 & Met.ext (abcde) $\rightarrow$ Met (abcde) \\
\hline R64 & Lys.ext (abcdef) $\rightarrow$ Lys (abcdef) \\
\hline R65 & His.ext (abcdef) $\rightarrow$ His (abcdef) \\
\hline R66 & Arg.ext (abcdef) $\leftrightarrow \operatorname{Arg}($ abcdef) \\
\hline
\end{tabular}

Suffix abbreviations: mitochondrial (.m), cytosolic (.c), extracellular (.ext), sink (.snk). Sink pools were used for metabolites that could not be balanced (Cys, Urea)

Balanced metabolite pools: 3PG, AKG, AcCoA.m, Ala, Arg, Asn, Asp, CO2, C1, Cit, Cys, DHAP, E4P, F6P, FBP, Fum, G6P, GAP, Gln, Glu, Gly, His, Ile, Lac, Leu, Lys, Mal, Met, OAC, P5P, PEP, Phe, Pro, Pyr.c, Pyr.m, S7P, Ser, Suc, SucCoA, Thr, Tyr, Val

Unbalanced metabolite pools: AcCoA.c, Ala.ext, Arg.ext, Asn.ext, Asp.ext, CO2.ext, Cit.ext, Cys.snk, Gln.ext, Glu.ext, Glc.ext, Gly.ext, His.ext, Ile.ext, Lac.ext, Leu.ext, Lys.ext, Met.ext, Phe.ext, Pro.ext, Pyr.ext, Ser.ext, Thr.ext, Tyr.ext, Urea.snk, Val.ext

a qui-square statistical test to evaluate goodness-of-fit. $95 \%$ confidence intervals of estimated fluxes were calculated through the parameter continuation method described by [3]. The number of degrees of freedom (n-p) associated with each flux estimation solution is 86 and 73 for NSCs and astrocytes, respectively (see supplementary material, Table S3). This means that in each case the number of independent measurements $\mathrm{n}$ (MIDs and extracellular fluxes) is superior to the number of free parameters $\mathrm{p}$ that were estimated (pools and unconstrained fluxes). A redundancy analysis is automatically performed during flux estimation by INCA, as described in [3]. Data overfitting was avoided by minimizing the number of metabolic reactions up to that necessary to accurately represent cellular carbon flow and satisfactorily simulate the data (i.e. intracellular compartmentation was considered only for pyruvate and no dilution fluxes or other pseudo-fluxes were used).

\section{Results and Discussion}

To study the metabolic modulations occurring during differentiation of mESC-derived NSCs to astrocytes, adherent monolayers of either fully-differentiated astrocytes or of NSCs were incubated with $\left[1-{ }^{13} \mathrm{C}\right]$ glucose. Samples from the culture supernatant and cell extracts were collected during $24 \mathrm{~h}$ (Figure 1a). During this period, cell viability was always above $90 \%$, and the exact protein amounts and cell number were determined for each individual dish used in the fluxomics study (data not shown). Astrocytes are larger in size when comparing to NSCs; they had on 
A

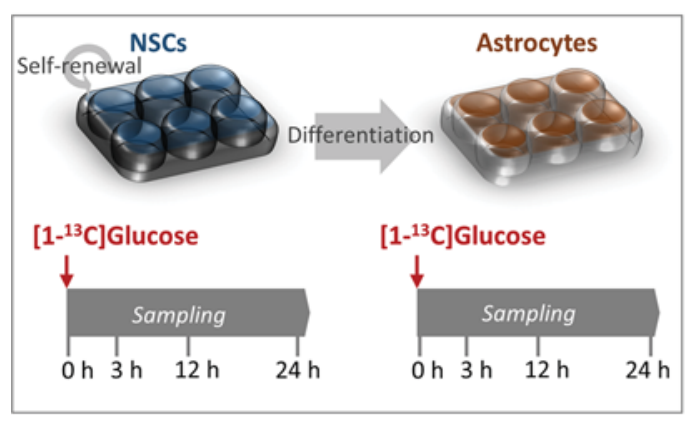

B

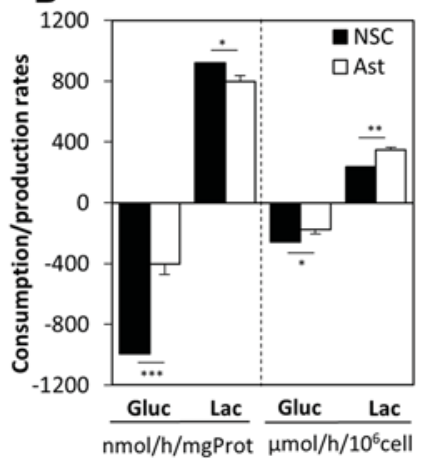

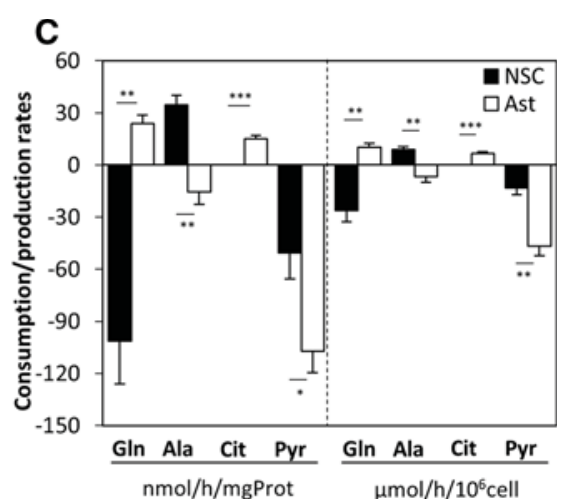

Fig. 1 a Schematic representation of the experimental design, consisting in the incubation of NSCs and NSC-derived astrocytes (Ast) with $\left[1-{ }^{13} \mathrm{C}\right]$ glucose. Consumption/production rates of $\mathbf{b}$ glucose and lactate, and $\mathbf{c}$ glutamine, alanine, citrate and pyruvate for NSCs and astrocytes. Values represent average rates (in $\mathrm{nmol} / \mathrm{mgProt} / \mathrm{h}$ or

average a 1.7-fold higher protein content than their undifferentiated counterparts.

\section{Modulation of Consumption and Production Rates During Astrocytic Differentiation}

Analysis of changes in supernatant composition of NSCs indicated a lactate production to glucose consumption ratio of approximately 1 (Figure $1 \mathrm{~b}$ ). Such high glycolytic metabolism in spite of oxygen availability is common to many stem cells [13, 42] and has been reported in proliferating NSCs [8]. It allows more metabolic intermediates to be available for the biosynthesis of cellular building blocks, which are required for proliferation [34]. Upon differentiation of NSCs into astrocytes, the glucose consumption decreased 1.5 -fold on a per cell basis (2.5-fold on a per $\mathrm{mg}$ of protein basis; Figure 1b). We calculated the consumption/production rates on a per cell and on a per $\mathrm{mg}$ of protein basis (Figure 1b, c) because both data normalizations are commonly found in brain metabolic studies and/or differentiation studies. Owing to the differences in cell size between NSCs and astrocytes, data interpretation is affected by the normalization method; for instance, while lactate production slightly decreases $(13 \%)$ on a per mg of protein basis, it is 1.5 -fold upregulated on a per cell basis upon differentiation. Nevertheless, the downregulation of glucose consumption was not matched by a proportional decrease in lactate production upon astrocytic differentiation, resulting in an even higher lactate production to glucose consumption ratio (close to 2). This extremely high glycolytic metabolism has been previously reported in primary cultures of cortical astrocytes (e.g. [2, 6]).

Striking differences between NSCs and astrocytes were also observed in the specific transport rates of some amino and organic acids (Figure 1c, Table S1). NSCs consumed glutamine at a high rate (10\% of the glucose uptake rate) $\mu \mathrm{mol} / 10^{6} \mathrm{cell} / \mathrm{h}$ as indicated) and error bars represent the standard deviation of biological replicates. Statistical significance was determined by performing a two-tailed Student t-test. Asterisks (*, ** and $* * *)$ denote statistically significant differences between conditions ( $\mathrm{p} \leq 0.1, \mathrm{p} \leq 0.05$ and $\mathrm{p} \leq 0.01$, respectively)

and, as opposed to astrocytes, cannot survive in glutaminefree medium (data not shown). Yeo et al. had already demonstrated the importance of the glutaminolysis pathway for long-term maintenance of NSCs [39]. Our comparative study showed that the NSC glutamine dependence is no longer present after its differentiation into astrocytes. NSC-derived astrocytes secreted glutamine (the rate was higher when cultured in glutamine-free medium), showing that even in the absence of neurons, these cells are able to recapitulate an important feature of the glutamate-glutamine cycle between neurons and astrocytes in the brain [5, 19]. Noteworthy, astrocytes took up glutamate from the medium at a similar rate of glutamine secretion (Table S1).

Another significant difference observed between these cell types was the upregulation of the pyruvate uptake rate upon astrocytic differentiation (3.6-fold on a per cell basis). Finally, astrocytes released citrate to the medium, while this metabolite was not detected in the medium of NSC cultures. Citrate secretion has been observed in primary cultures of astrocytes, but the reason is not entirely understood [36, 37, 43].

\section{Intracellular ${ }^{13} \mathrm{C}$-labelling in NSCs and Astrocytes}

After label administration, the fraction of $\left[1-{ }^{13} \mathrm{C}\right]$ glucose measured intracellularly by GC-MS was $87 \%$ in NSCs and $84 \%$ in astrocytes. In both cell populations, the label enrichment of the glycolytic intermediates 3PG and PEP reached values over $40 \%$, showing conservation of the ${ }^{13} \mathrm{C}$ label coming from glucose (Figure 2). Since the first carbon of glucose is lost as $\mathrm{CO}_{2}$ in the oxidative branch of the PPP, these results indicate that the non-oxidative PPP branch had low activity in both cell populations. Overall, the label enrichment in lactate, alanine, TCA cycle intermediates and related amino acids was lower than in 


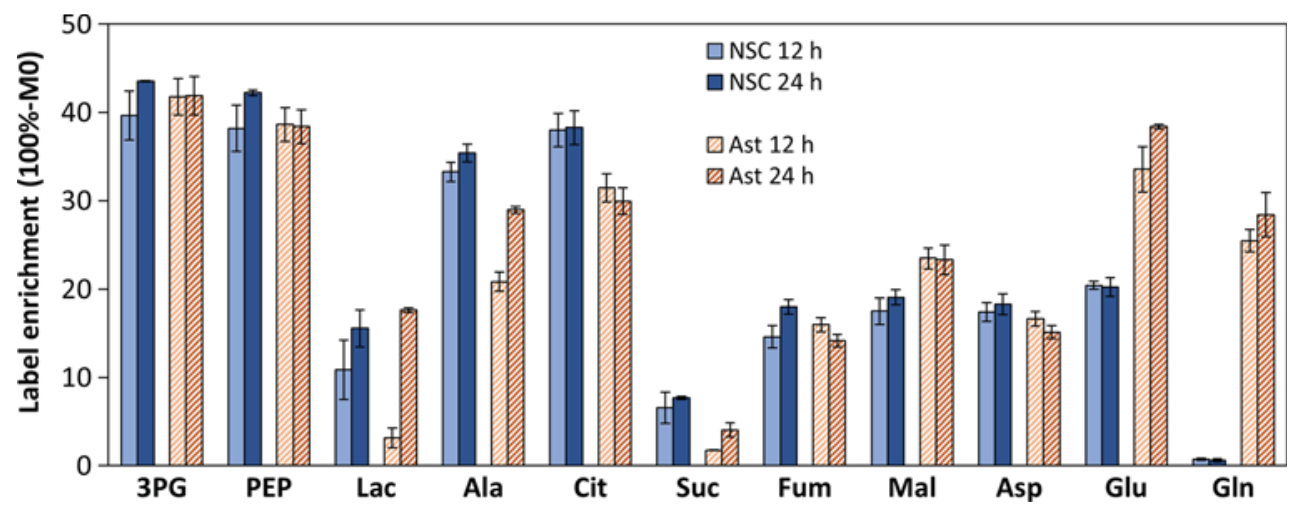

Fig. 2 Label enrichment of intracellular metabolites at $12 \mathrm{~h}$ and $24 \mathrm{~h}$ after $\left[1-{ }^{13} \mathrm{C}\right]$ glucose addition to NSCs and astrocytes. The values correspond to the sum of all labeled isotopomers (100\%-M0). Error bars represent the standard deviation of biological replicates. Shown

metabolites: 3-phosphoglyceric acid (3PG), phosphoenolpyruvate (PEP), lactate (Lac), alanine (Ala), citrate (Cit), succinate (Suc), fumarate (Fum), malate (Mal), aspartate (Asp), glutamate (Glu) and glutamine (Gln)

glycolytic metabolites, indicating the contribution of other carbon sources (Figure 2).

Despite the similarities, several differences emerged in the intracellular labelling patterns of NSCs and astrocytes. Firstly, isotopic steady-state was achieved for most metabolites in NSCs within $24 \mathrm{~h}$, but not in astrocytes (Figures 3 and 4), in agreement with the downregulation of astrocytic glucose consumption. The largest difference in the rate of ${ }^{13} \mathrm{C}$ incorporation between both populations was observed for the lactate pool at $12 \mathrm{~h}$ post label addition. The M1 isotopomer abundance was $11 \%$ in NSCs while it was only $3 \%$ in astrocytes. The higher consumption of (unlabeled) pyruvate from the medium by

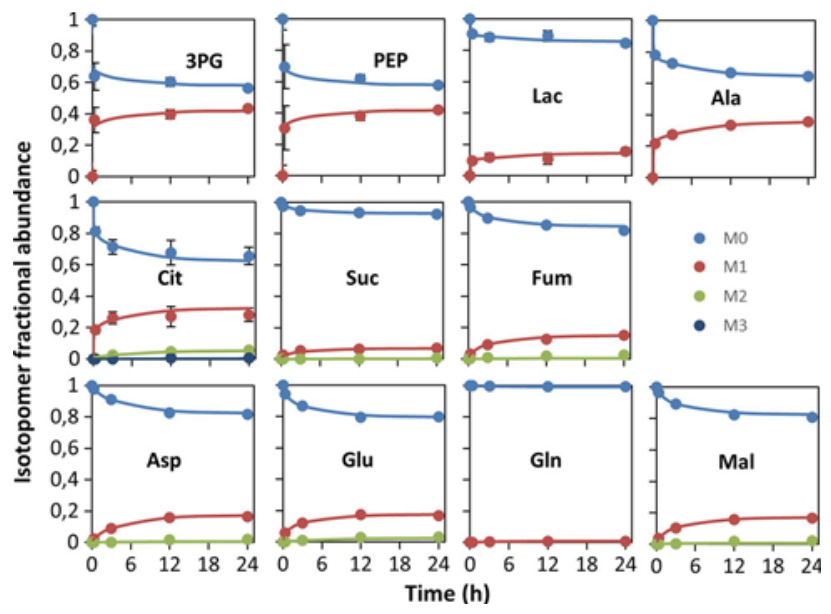

Fig. 3 Intracellular ${ }^{13} \mathrm{C}$-labelling dynamics along $24 \mathrm{~h}$ culture of NSCs in the presence of $\left[1-{ }^{13} \mathrm{C}\right]$ glucose. Symbols correspond to GC-MS measurements corrected for natural isotope abundance. Error bars represent the standard deviation of biological replicates. Lines correspond to model fits obtained by non-stationary ${ }^{13} \mathrm{C}$-MFA. M0 denotes the parent fragment with a certain ion mass/charge ratio and $\mathrm{Mm}$ denotes the increased mass/charge ratio due to $\mathrm{m}$ labelled ${ }^{13} \mathrm{C}$ atoms. M0, M1, M2 and M3 measured values are provided in Table S2 astrocytes may have contributed to the slower labelling of the astrocytic lactate pool. Moreover, NSCs displayed higher labelling in the citrate $(38 \%$ vs. $30 \%)$ and succinate ( $8 \%$ vs. $4 \%$ ) pools compared with astrocytes, but this difference was not propagated throughout the TCA cycle. In fact, the opposite result was observed for the malate pool, which was consistently more highly ${ }^{13} \mathrm{C}$ enriched in astrocytes at $12 \mathrm{~h}$ and $24 \mathrm{~h}$ after label addition. Moreover, succinate was less labeled than fumarate (57\% less in NSCs and $71 \%$ less in astrocytic populations), which suggests additional routes of label entry into the TCA cycle, and at the same time suggests that succinate dehydrogenase (SucD) has limited reverse

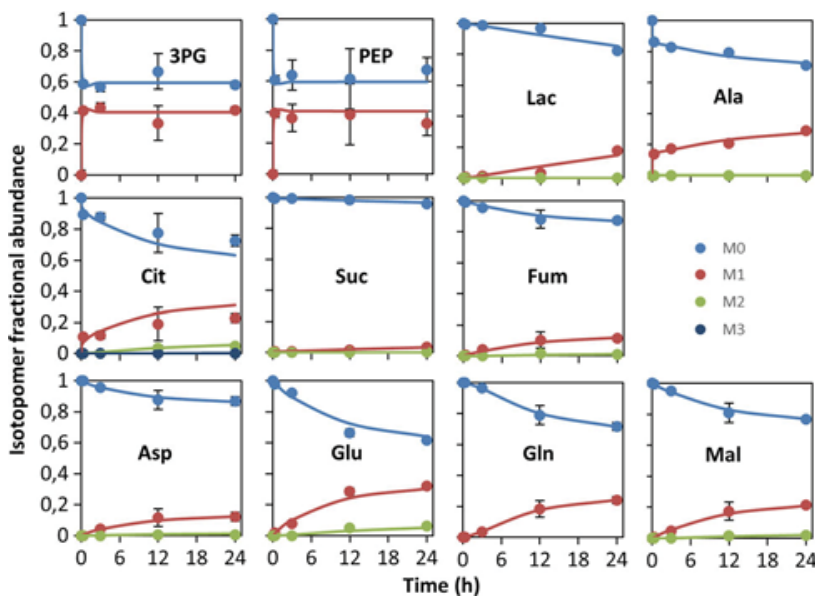

Fig. 4 Intracellular ${ }^{13} \mathrm{C}$-labelling dynamics along $24 \mathrm{~h}$ culture of astrocytes in the presence of $\left[1-{ }^{13} \mathrm{C}\right]$ glucose. Symbols correspond to GC-MS measurements corrected for natural isotope abundance. Error bars represent the standard deviation of biological replicates. Lines correspond to model fits obtained by non-stationary ${ }^{13} \mathrm{C}$-MFA flux estimation. M0 denotes the parent fragment with a certain ion mass/ charge ratio and $\mathrm{Mm}$ denotes the increased mass/charge ratio due to $\mathrm{m}$ labelled ${ }^{13} \mathrm{C}$ atoms. M0, M1, M2 and M3 measured values are provided in Table $\mathrm{S} 2$ 
activity. Finally, significant enrichment in intracellular glutamine was observed in astrocytes but not in NSCs, consistent with the measured glutamine secretion and consumption rates, respectively. However, when cultured in glutamine-free media, NSCs also incorporated significant ${ }^{13} \mathrm{C}$ from glucose into glutamine despite the viability loss, indicating glutamine synthetase (GS) activity (data not shown).

\section{Fluxome Rearrangements During Astrocytic Differentiation of NSCs}

Using isotopic non-stationary ${ }^{13} \mathrm{C}$-MFA, metabolic fluxes were estimated for each cell type by integrating the timeprofiles of the mass isotopomer distributions (Figures 3, 4) with extracellular transport rates (Table S1) in a metabolic network model (Table 1). Reasonably good fits were obtained for all metabolites in both cultures (Figures 3, 4). All estimated fluxes and associated $95 \%$ confidence intervals are provided as Supporting Information (Table S3).

For a global comparison of metabolic differences between NSCs and astrocytes, metabolic flux ratios were calculated for fluxes with finite lower and upper confidence interval bounds which exclude the value zero (Figure 5a). Generally, NSCs displayed a higher metabolic flux in central carbon metabolism compared with astrocytes, including glycolysis (1.7-fold) and the TCA cycle (e.g. 19.4-fold higher citrate synthase flux). A detailed view of these differences can be observed in Fig. 5b, where the thickness and color of arrows reflect absolute flux values. These flux maps show that the activity of the PPP remained lower than $6 \%$ of the corresponding glycolytic flux in both cell populations. Half of the cytosolic pyruvate was converted to mitochondrial pyruvate in NSCs, while in astrocytes most of the cytosolic pyruvate was diverted to lactate secretion, consistent with the measured extracellular rates. In mitochondria, the majority of pyruvate in NSCs entered the TCA cycle by conversion to Acetyl-CoA (AcCoA) through pyruvate dehydrogenase activity (PDH; 318.0 $\mathrm{nmol} / \mathrm{h} / 10^{6}$ cell), and the rest was carboxylated to oxaloacetate $(\mathrm{OAC})\left(\mathrm{PC} ; 57.7 \mathrm{nmol} / \mathrm{h} / 10^{6}\right.$ cell), resulting in a $\mathrm{PC} / \mathrm{PDH}$ ratio of 0.18 . On the other hand, astrocytes metabolization of mitochondrial pyruvate through $\mathrm{PC}$ was 4.3-fold higher $\left(39.3 \mathrm{nmol} / \mathrm{h} / 10^{6}\right.$ cell) than through $\mathrm{PDH}$ (9.1 nmol/h/10 $60^{6}$ cell). This anaplerotic metabolization of pyruvate in astrocytes contributed to support glutamine and citrate secretion. In contrast, glutamine was taken up from the medium by NSCs and metabolized to citrate via reductive carboxylation of alpha-ketoglutarate (AKG). The importance of this metabolic route was first identified in

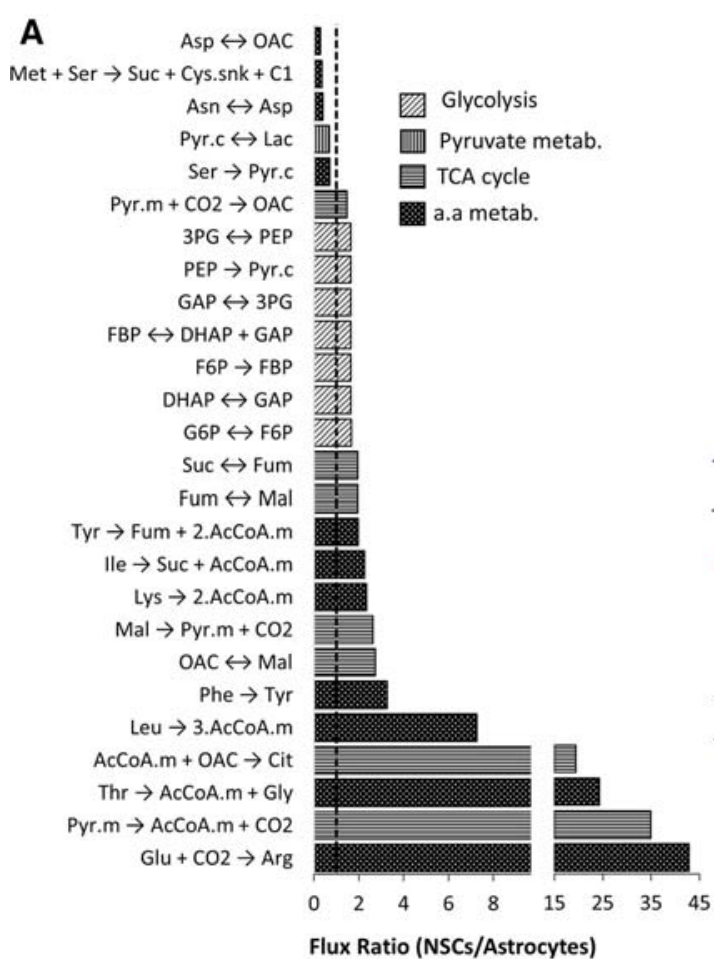

Fig. 5 Overview of metabolic flux distributions of NSCs and astrocytes. a Metabolic flux ratios between cultures of NSCs and astrocytes. The estimated fluxes considered have finite lower and upper $95 \%$ confidence interval bounds that exclude zero. b Metabolic

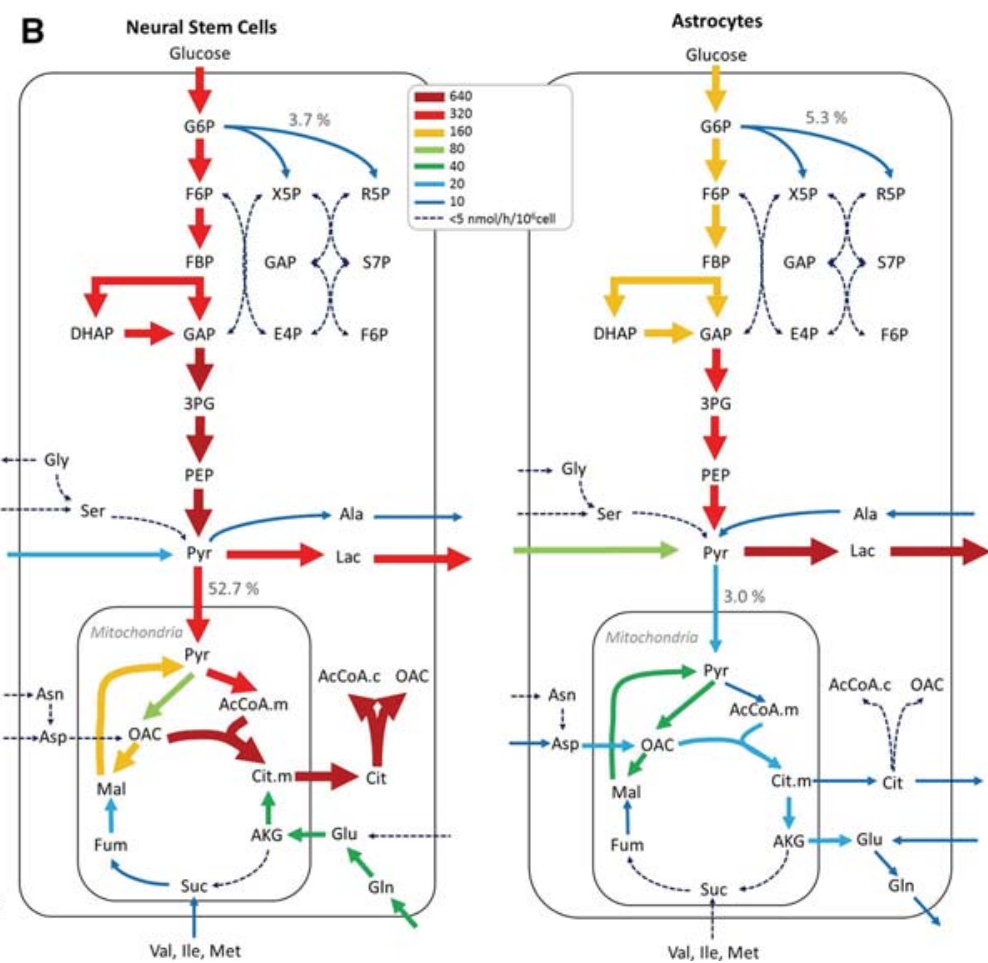

flux maps for NSCs and astrocytes. Arrow thickness and colors reflect flux values in units of $\mu \mathrm{mol} / \mathrm{h} / 10^{6}$ cell (see Table S3 for exact flux values and associated $95 \%$ confidence intervals) 
cancer cell cultures to support lipogenesis [20], and a recent study suggests its activity also in human NSCs [24]. Although not pointed out by the authors, the prominent abundance of citrate M5 isotopomer upon incubation with $\left[\mathrm{U}_{-}{ }^{13} \mathrm{C}\right]$ glutamine is indicative of reductive carboxylation of glutamine-derived AKG. This reaction involves addition of an unlabelled carbon by isocitrate dehydrogenase (IDH) acting in reverse relative to the canonical oxidative TCA cycle, which produces citrate M4 from $\left[\mathrm{U}^{-13} \mathrm{C}\right]$ glutamine. In line with these results, [17] showed that de novo lipogenesis is important for NSCs proliferation. These cells have high enzymatic activity of fatty acid synthase (Fasn) and the inhibition of Fasn led to a significant reduction in proliferation.

Finally, a net flux of malate formation from OAC was estimated in both cell populations, which was then recycled back to pyruvate by malic enzyme (ME). Regardless of the total pyruvate flux that enters the TCA cycle, pyruvate cycling becomes rather important through the activity of $\mathrm{ME}$ and PC to balance TCA cycle activity and biosynthesis efflux in both NSCs and astrocytes.

\section{Conclusion}

A deeper understanding of the metabolic circuits in NSCs and how they evolve during differentiation may provide novel approaches for reactivating astrogenesis and neurogenesis to treat neurodegenerative diseases. Herein, we aimed at assessing the alterations in central carbon metabolism associated with the differentiation of NSCs into astrocytes using non-stationary ${ }^{13} \mathrm{C}$-MFA. This is the first study in which a comparison of metabolic fluxes between these cell populations was performed, highlighting significant differences at the level of glycolysis, TCA cycle and amino acids metabolism. Specifically, astrocytic differentiation is followed by a downregulation of glucose consumption, but astrocytes keep an even higher lactate production to glucose consumption ratio than NSCs. Moreover, NSCs generated citrate by reductive carboxylation of AKG and channeled it towards cytosolic AcCoA formation, a precursor for fatty acid biosynthesis; in turn, the TCA cycle of astrocytes was rewired to support citrate and glutamine secretion, both metabolic features typically reported in primary astrocytic cultures. While the $\left[1-{ }^{13} \mathrm{C}\right]-$ glucose tracer used in this study provided a first overview of central carbon metabolism in this compelling biological setting, parallel experiments with other labeled substrates should be performed to specifically probe some of the identified metabolic features. For instance, [U- $\left.{ }^{13} \mathrm{C}\right] \mathrm{glu}-$ tamine could be used to confirm the prominent reductive carboxylation flux in NSCs. Due to its power to resolve intracellular fluxes, we expect the multiplication of ${ }^{13} \mathrm{C}$ MFA studies of neural metabolism in the future.

Acknowledgments Support from iNOVA4Health - UID/Multi/ 04462/2013, a program funded by Fundação para a Ciência e a Tecnologia (FCT)/Ministério da Educação e Ciência and co-funded by FEDER under the PT2020 Partnership Agreement, is acknowledged. This research has also received support from FCT through the project MITP-TB/ECE/0013/2013, from the German research foundation (RTG1331, KoRS-CB) and the German ministry for science (BMBF-DynaMeTox). JV Sá is a recipient of a Ph.D. fellowship from FCT (PD/BD/52474/2014). The expert technical assistance of Lars Evje with GC-MS is gratefully acknowledged. We are also thankful to Nuno Carinhas for his help on fluxome analysis.

\section{References}

1. Ahn WS, Antoniewicz MR (2013) Parallel labeling experiments with [1,2-(13)C]glucose and [U-(13)C]glutamine provide new insights into CHO cell metabolism. Metabolic Engineering 15:34-47

2. Amaral AI, Teixeira AP, Håkonsen BI, Sonnewald U, Alves PM (2011) A comprehensive metabolic profile of cultured astrocytes using isotopic transient metabolic flux analysis and C-labeled glucose. Frontiers in Neuroenergetics $3: 1-5$

3. Antoniewicz MR, Kelleher JK, Stephanopoulos G (2006) Determination of confidence intervals of metabolic fluxes estimated from stable isotope measurements. Metabolic Engineering 8(4):324-337

4. Bak LK, Schousboe A, Waagepetersen HS (2006) The glutamate/ GABA-glutamine cycle: Aspects of transport, neurotransmitter homeostasis and ammonia transfer. Journal of Neurochemistry 98(3):641-653

5. Bélanger M, Allaman I, Magistretti PJ (2011) Brain energy metabolism: focus on astrocyte-neuron metabolic cooperation. Cell Metabolism 14(6):724-738

6. Bolanos JP, Peuchen S, Heales SJR, Land JM, Clark JB (1994) Nitric oxide-mediated inhibition of the mitochondrial respiratory chain in cultured astrocytes. Journal of Neurochemistry 63:910-916

7. Brown AM (2004) Brain glycogen re-awakened. Journal of Neurochemistry 89(3):537-552

8. Candelario KM, Shuttleworth CW, Cunningham LA (2013) Neural stem/progenitor cells display a low requirement for oxidative metabolism independent of hypoxia inducible factor-1 alpha expression. Journal of Neurochemistry 125(3):420-429

9. Carinhas N, Bernal V, Monteiro F, Carrondo MJT, Oliveira R, Alves PM (2010) Improving baculovirus production at high cell density through manipulation of energy metabolism. Metabolic Engineering 12(1):39-52

10. Carinhas N, Pais DAM, Koshkin A, Fernandes P, Coroadinha A, Carrondo MJT, Alves PM, Teixeira AP (2016) Metabolic flux profiling of MDCK cells during growth and canine adenovirus vector production. Scientific Reports 6:23529

11. Crown SB, Antoniewicz MR (2013) Publishing (13)C metabolic flux analysis studies: A review and future perspectives. Metabolic Engineering 20:42-48

12. Doetsch F, Caille I, Lim DA, Garc1 JM, Alvarez-buylla A (1999) Subventricular Zone Astrocytes Are Neural Stem Cells in the Adult Mammalian Brain. Cell 97:703-716

13. Folmes CDL, Nelson TJ, Martinez-Fernandez A, Arrell DK, Lindor JZ, Dzeja PP, Ikeda Y, Terzic CP, Terzic A (2011) Somatic oxidative bioenergetics transitions into pluripotency- 
dependent glycolysis to facilitate nuclear reprogramming. Cell Metabolism 14(2):264-271

14. Hofmann U, Maier K, Niebel A, Vacun G, Reuss M, Mauch K (2008) Identification of metabolic fluxes in hepatic cells from transient 13C-labeling experiments: Part I. Experimental observations. Biotechnology and Bioengineering 100(2):344-354

15. Johnson MA, Weick JP, Pearce RA, Zhang S-C (2007) Functional Neural Development from Human Embryonic Stem Cells: Accelerated Synaptic Activity Coculture. Journal of Neuroscience 27(12):3069-3077

16. Kleiderman SM, Sá JV, Teixeira AP, Brito C, Gutbier S, Evje LG, Hadera MG, Glaab E, Henry M, Sachinidis A, Alves PM, Sonnewald U, Leist M (2016) Functional and phenotypic differences of pure populations of stem cell-derived astrocytes and neuronal precursor cells. Glia 64(5):695-715

17. Knobloch M, Braun SMG, Zurkirchen L, von Schoultz C, Zamboni N, Araúzo-Bravo MJ, Kovacs WJ, Karalay Ö, Suter U, Machado RAC, Roccio M, Lutolf MP, Semenkovich CF, Jessberger S (2013) Metabolic control of adult neural stem cell activity by Fasn-dependent lipogenesis. Nature 493(7431):226-230

18. Kriegstein A, Alvarez-Buylla A (2009) The glial nature of embryonic and adult neural stem cells. Annual Review of Neuroscience 32:149-184

19. McKenna M, Gruetter R, Sonnewald U, Waagepetersen H, Schousboe A (2012) Energy Metabolism of the Brain. In: Brady STS, Albers RW, Price DL (eds) Basic Neurochemistry: Principles of Molecular, Cellular, and Medical Neurobiology, 8th edn. Elsevier Academic Press, Oxford, UK, pp 200-299

20. Metallo CM, Gameiro PA, Bell EL, Mattaini KR, Yang J, Hiller K, Jewell CM, Johnson ZR, Irvine DJ, Guarente L, Kelleher JK, Vander Heiden MG, Iliopoulos O, Stephanopoulos G (2012) Reductive glutamine metabolism by IDH1 mediates lipogenesis under hypoxia. Nature 481:380-384

21. Munger J, Bennett BD, Parikh A, Feng X, Rabitz HA, Shenk T, Rabinowitz JD (2008) Systems-level metabolic flux profiling identifies fatty acid synthesis as a target for antiviral therapy. Nat Biotechnol 26(10):1179-1186

22. Nedergaard M, Ransom B, Goldman SA (2003) New roles for astrocytes: Redefining the functional architecture of the brain. Trends in Neurosciences 26(10):523-530

23. Noh K, Wiechert W (2006) Experimental Design Principles for Isotopically Instationary C Labeling Experiments. Biotechnology and Bioengineering 94:234-251

24. Palm T, Bolognin S, Meiser J, Nickels S, Träger C, Meilenbrock R-L, Brockhaus J, Schreitmüller M, Missler M, Schwamborn JC (2015) Rapid and robust generation of long-term self-renewing human neural stem cells with the ability to generate mature astroglia. Scientific Reports 5:16321

25. Pellerin L, Bouzier-Sore A-K, Aubert A, Serres S, Merle M, Costalat R, Magistretti PJ (2007) Activity-Dependent Regulation of Energy Metabolism by Astrocytes: An Update. Glia 55:1251-1262

26. Pellerin L, Magistretti PJ (1994) Glutamate uptake into astrocytes stimulates aerobic glycolysis: a mechanism coupling neuronal activity to glucose utilization. Proceedings of the National Academy of Sciences of the United States of America 91(22): 10625-10629
27. Phatnani H, Maniatis T (2015) Astrocytes in Neurodegenerative Disease. Cold Spring Harbor Perspectives in Biology 7(6):a020628

28. Rouach N, Koulakoff A, Abudara V, Willecke K, Giaume C (2008) Astroglial Metabolic Networks Sustain Hippocampal Synaptic Transmission. Science 322:1551-1555

29. Sá, J. V., Duarte, T. M., Carrondo, M. J. T., Alves, P. M., and Teixeira, A. P. (2015). Metabolic Flux Analysis: A Powerful Tool in Animal Cell Culture. In M. Al-Rubeai (Ed.), Animal Cell Culture (Vol. 521-539, p. 785). Springer International Publishing

30. Sauer U (2006) Metabolic networks in motion: 13C-based flux analysis. Molecular Systems Biology. doi:10.1038/msb4100109

31. Seri B, Garc1 JM, Mcewen BS, Alvarez-buylla A (2001) Astrocytes Give Rise to New Neurons in the Adult Mammalian Hippocampus. The Journal of Neuroscience 21(18):7153-7160

32. Simard M, Nedergaard M (2004) The neurobiology of glia in the context of water and ion homeostasis. Neuroscience 129(4):877-896

33. Ullian, E. M., Sapperstein, S. K., Christopherson, K. S., and Barres, B. a. (2001). Control of synapse number by glia. Science (New York, N.Y.), 291, 657-661

34. Vander Heiden MG, Cantley LC, Thompson CB (2009) Understanding the Warburg Effect : Cell Proliferation. Science 324:1029-1034

35. Walls, A. B., Bak, L. K., Sonnewald, U., Schousboe, A., and Waagepetersen, H. S. (2014). Metabolic Mapping of Astrocytes and Neurons in Culture Using Stable Isotopes and Gas Chromatography-Mass Spectrometry (GC-MS). In HirrlingerJohannes \& H. S. Waagepetersen (Eds.), Brain Energy Metabolism (pp. 73-105)

36. Westergaard, N., Banke, T. U. E., Wahl, P., Sonnewaldt, U., and Schousboe, A. (1995). Citrate modulates the regulation by $\mathrm{Zn} 2+$ of N-methyl-D-aspartate receptor-mediated channel current and neurotransmitter release, 92(April), 3367-3370

37. Westergaard N, Sonnewald U, Unsgård G, Peng L, Hertz L, Schousboe A (1994) Uptake, release, and metabolism of citrate in neurons and astrocytes in primary cultures. Journal of Neurochemistry 62(5):1727-1733

38. Wiechert W (2001) 13C metabolic flux analysis. Metabolic Engineering 3(3):195-206

39. Yeo H, Lyssiotis CA, Zhang Y, Ying H, Asara JM, Cantley LC, Paik J-H (2013) FoxO3 coordinates metabolic pathways to maintain redox balance in neural stem cells. The EMBO Journal 32(19):2589-2602

40. Young, J. D. (2014). INCA: A computational platform for isotopically nonstationary metabolic flux analysis. Bioinformatics (Oxford, England), 30, 11-13

41. Young JD, Walther JL, Antoniewicz MR, Yoo H, Stephanopoulos G (2008) An elementary metabolite unit (EMU) based method of isotopically nonstationary flux analysis. Biotechnology and Bioengineering 99(3):686-699

42. Zhang J, Nuebel E, Daley GQ, Koehler CM, Teitell MA (2012) Metabolic regulation in pluripotent stem cells during reprogramming and self-renewal. Cell Stem Cell 11(5):589-595

43. Zwingmann C, Leibfritz D (2003) Regulation of glial metabolism studied by 13C-NMR. NMR in Biomedicine 16(6-7):370-399 